\section{KNOWING THE COST OF \\ CARDIAC SURGICAL \\ COMPLICATIONS: DOES IT \\ REALLY REDUCE THEM OR \\ IMPROVE QUALITY? \\ To the Editor:}

I read with interest the article by Christensen and colleagues ${ }^{1}$ about the incremental costs of postoperative hemorrhage in cardiac surgery, a common complication. I agree with the authors that measures to prevent or decrease postoperative hemorrhage are important in cost-effectiveness. In fact, all cardiac surgical complications are associated with substantial added cost, as shown in several articles. ${ }^{2}$

Cardiac surgery has its own special peculiarities that make it different from any other kind of surgery. It has the widest spectrum of complications, most of them are fatal. With regard to the etiology of postoperative hemorrhage, a great deal is attributable to the vast array of antiplatelet and thrombolytic agents given before surgery by referring cardiologists. Most other causes mentioned by Christensen and colleagues ${ }^{1}$ are usually unavoidable.

It is difficult to calculate the exact cost of each individual complication, because complications usually start as one kind and rapidly move on to another in a cascade pattern, ending this vicious circle with multiorgan failure and death. Postoperative renal failure necessitating hemodialysis, prolonged need for ventilation, and mediastinitis are among the most ex-

\footnotetext{
The Editor welcomes submissions for possible publication in the Letters to the Editor section that consist of commentary on an article published in the Journal or other relevant issues. Authors should: • Include no more than 500 words of text, three authors, and five references. • Type with double-spacing. • See http://jics.ctsnetjournals.org/misc/ifora.shtml for detailed submission instructions. - Submit the letter electronically via jtcvs.editorialmanager.com. Letters commenting on an article published in the JTCVS will be considered if they are received within 6 weeks of the time the article was published. Authors of the article being commented on will be given an opportunity of offer a timely response ( 2 weeks) to the letter. Authors of letters will be notified that the letter has been received. Unpublished letters cannot be returned.
}

pensive complications, by far exceeding the cost of bleeding. All these complications deserve preventive measures, but because of their harmful effects on the patients rather than for cost containment.

Avoiding complications is much better than handling and overcoming them. Careful selection of patients who will benefit most with the least trouble is the criterion standard of the ideal patient. Quality improvement in this multidisciplinary and delicate service requires a comprehensive understanding of the basics and details of the surgical procedures, not the incremental costs of complications. Morbidity and mortality can not be "priced" financially, because there is no winner in this deal. Both the patient and the health service are losers. Knowing the added cost will never reduce the complications. Death, the most disastrous complication, is actually the cheapest, with no extra cost to be paid. In a series from my own group, ${ }^{3}$ lifelong hemodialysis was the most expensive complication, followed by a patient being "stuck" on the ventilator.

The most important feature of cardiac surgery is that only a certain subset of cardiac patients, according to clinical guidelines, will benefit from surgery. All cardiac procedures, except patent ductus arteriosus ligation, are palliative. The ongoing atherosclerosis progression will sooner or later "block" the grafts. Valve replacement, as the name implies, actually replaces one disease condition with another. The concept of "do no harm" must be exercised with every cardiac patient. Health care providers must avoid complications because of this concept, not because of extra cost. Candidates at very high risk who will receive only marginal surgical benefit should not undergo surgery. Cardiac patients must be considered according to the indication and type of surgical procedure and assessed according to the international risk stratification scores. Strict adherence to evidence-based medicine must be reinforced and practiced by the referring cardiologist, cardiac anesthetist, perfusionist, surgical team, and intensive care staff. A successful cardiac procedure is the outcome of perfect performance by all these team members in conjunction with the previously mentioned patient factors.

\section{Khaled Al-Ebrahim, FRCSC Department of Cardiac Surgery University Hospital Jeddah, Saudi Arabia}

\section{References}

1. Christensen MC, Krapf S, Kempel A, Heymann C Costs of excessive postoperative hemorrhage in cardiac surgery. J Thorac Cardiovasc Surg. 2009;138: 687-3.

2. Speir AM, Kasirajan V, Barnett SD, Fonner E Jr. Additive costs of complications for isolated coronary artery bypass grafting patients in Virginia. Ann Thorac Surg. 2009;88:40-6.

3. Al-Ebrahim KE, Tahir MZ, Rustom M, Shafei M. Cardiac surgery in patients with impaired renal function. J King Abdulaziz University Med Sci. 1994;4:49-53.

doi:10.1016/j.jtcvs.2009.08.064

\section{Reply to the Editor:}

We naturally agree with $\mathrm{Dr} \mathrm{Al}-$ Ebrahim that avoiding complications in cardiac surgery is far better than treating them as they occur; however, we believe that some key messages of our research have been misunderstood. Dr Al-Ebrahim states, "Quality improvement in this multidisciplinary and delicate service requires comprehensive understanding of the basics and details of the surgical procedures, and the incremental costs of complications. Morbidity and mortality can not be "priced" financially, because there is no winner in this deal. Both the patient and the health service are losers. Knowing the added cost will never reduce the complications. Death, the most disastrous complication, is actually the cheapest, with no extra cost to be paid." Although it is correct that morbidity and mortality cannot be priced, the costs of treating medical 
complications and even of saving lives can certainly be estimated. ${ }^{1}$ Indeed, the very cornerstone of the health economic discipline is to do exactly such estimations across the most common diseases to determine how limited health care resources can be used most effectively to benefit the most patients. Knowing the economic consequences of medical complications is a highly essential step in our efforts to identify cost-effective clinical interventions to address them. In health care systems with limited resources, policy makers, health insurers, hospital administrators, and practicing physicians need to consider carefully the cost-effectiveness of medical interventions, both in the daily practice of medicine and as part of long-term evaluation and planning of quality improvement initiatives.

As we describe in our article, medical interventions can either be cost saving (if the hospital's cost savings are greater than the cost of the intervention), cost-effective (when the incremental cost of a clinical intervention is reasonable with regard to the clinical benefits obtained), or cost enhancing (if the clinical intervention induces higher hospital costs without any clinical benefit). In the context of excessive hemorrhage in cardiac surgery, knowledge of the medical and economic consequences of this complication is the very first step in the identification of safe, effective, and cost-effective clinical interventions to address this complication. Additionally, awareness of the costs of excessive hemorrhage may compel health care professionals to apply preventive and therapeutic measures as early as possible. In our research we used simple and objective criteria for excessive postoperative hemorrhage to allow early identification of patients at risk for severe morbidity or even a fatal outcome. Early identification of bleeding facilitates early treatment and thereby improves outcome. This is the very basis for improvement in clinical care and applied quality improvement.
Of course, health care providers must primarily keep the individual patient's health in mind. In addition, however, we need to be aware of the costs of complications to provide the highest standard of care for all patients. This may be even more important in the future, when rationing of health care resources is likely to become an even more dominant part of our health care services.

\section{Michael C. Christensen, DrPH ${ }^{a}$} Stephan Krapf, $M D^{b}$ Angela Kempel, $\mathrm{MSc}^{c}$ Christian von Heymann, $M D$, $P h D, D E A A^{d}$

${ }^{a}$ Novo Nordisk A/S

Medical \& Science Haemostasis Department, Bagsvaerd, Denmark

${ }^{b}$ Klinik für Herzchirurgie Herzzentrum Augsburg Augsburg, Germany

${ }^{c}$ Pharmametrics $\mathrm{GmbH}$

Institute for Health Economics \& Epidemiology

Freiburg, Germany

${ }^{d}$ Department of Anaesthesiology and Intensive Care Medicine

Charité-University Medicine Berlin Campus Virchow-Klinikum Berlin, Germany

\section{Reference}

1. Dolan P, Metcalfe R, Munro V, Christensen MC. Valuing lives and life years: anomalies, implications, and an alternative. Health Econ Policy Law. 2008;3(Pt 3):277-300.

doi:10.1016/j.jtcvs.2010.02.009

\section{DOES PREVIOUS}

\section{PERCUTANEOUS CORONARY} INTERVENTION INCREASE THE RISK OF GRAFT FAILURE IN SUBSEQUENT CORONARY SURGERY?

\section{To the Editor:}

We recently pointed out ${ }^{1}$ that there is no evidence to support significantly better angiographic patency with radial artery conduit than with saphenous vein graft in coronary artery bypass grafting in controlled, randomized trials reported to date. We do not, however, support inclusion of data extracted from the article by Gaudino and associates ${ }^{2}$ in the recently published meta-analysis by Benedetto and colleagues ${ }^{3}$ of controlled, randomized trials comparing radial artery conduits and saphenous vein grafts with respect to angiographic patency.

The article by Gaudino and associates ${ }^{2}$ reported 2 controlled, randomized trials including patients with previous percutaneous stent implantation (in any coronary vessel) with preoperative angiographic confirmation of a failed (I trial) or patent (II trial) intracoronary stent. In that report, ${ }^{2}$ they focused on the results of arterial versus venous grafts directed to the first obtuse marginal artery. There were, however, no data on failed stent location. In a subsequent analysis ${ }^{4}$ of that initial report, Gaudino and coworkers ${ }^{4}$ focused on the complementary venous grafts to nonobtuse target coronary vessels (right coronary artery and circumflex artery other than the first obtuse marginal artery). They concluded that patients with development of in-stent restenosis face a higher risk of early venous graft failure (46 occluded of 84 , patency rate $45.2 \%$ at 5 years after surgery). Even in that article, however, there were no precise data about the number of failed venous grafts that were distributed to targeted coronary arteries with in-stent restenosis. We were able to find out only that 25 of the failed stents were located on a circumflex artery other than the first obtuse marginal artery, 31 were located on the right coronary artery, and 7 were located on the left anterior descending coronary artery. Although 43 venous graft-targeted vessels were circumflex artery other than the first obtuse marginal artery and 41 were right coronary artery, we must face the possibility that the vast majority of venous grafts were placed on previously stented coronary arteries.

It has been argued by Gaudino and associates $^{2,4}$ that in-stent restenosis is 